\title{
Quantification of the Radiographic Joint Space Width of the Ankle
}

\author{
Berna Goker MD, Emel Gonen MD, \\ Mehmet D. Demirag MD, Joel A. Block MD
}

Received: 25 June 2008/Accepted: 25 March 2009/Published online: 21 April 2009

(C) The Association of Bone and Joint Surgeons 2009

\begin{abstract}
Quantification of joint space width of the ankle could provide information essential to evaluate the effects of potential disease-modifying agents and adverse effects of devices intended to ameliorate osteoarthritis elsewhere in the lower extremity. Current methods require proprietary software or have not been well validated; our purpose was to develop and assess the reliability of a digital joint space width quantification method using public access software. We studied 95 patients, asymptomatic in the ankles and without history of ankle trauma, but with symptomatic medial knee osteoarthritis, participating in an ongoing longitudinal trial. Weightbearing anteroposterior radiographs of
\end{abstract}

One or more of the authors have received funding from the National Institutes of Health, Bethesda, MD (NIH 1P50 AR048941) (JAB) and the Turkish Society for Education and Research in Rheumatology (RAED-Romatoloji Arastırma ve Egitim Dernegi) (BG).

Each author certifies that his or her institution has approved the human protocol for this investigation, that all investigations were conducted in conformity with ethical principles of research, and that informed consent for participation in the study was obtained.

This work was performed at Gazi University and at Rush Medical College.

\section{B. Goker ( $₫)$, M. D. Demirag}

School of Medicine, Department of Internal Medicine, Section of Rheumatology, Gazi University, Besevler, Ankara 06500, Turkey

e-mail: bgoker@yahoo.com

\section{E. Gonen}

Department of Orthopaedics and Traumatology, Ankara Diskapi Research Hospital, Ankara, Turkey

\section{J. A. Block}

Departments of Biochemistry and Internal Medicine, Section of Rheumatology, Rush Medical College, Rush University Medical Center, 1725 W. Harrison Street, Suite 1017, Chicago, IL 60612, USA the ankle and supine radiographs of the pelvis were assessed, and the narrowest medial and lateral tibiotalar joint space widths and hip joint space widths were measured using Image J software (US NIH, Bethesda, MD). Medial joint space widths were $2.56 \pm 0.50$ and $2.55 \pm 0.48 \mathrm{~mm}$, and lateral joint space widths were $2.45 \pm 0.55$ and $2.44 \pm 0.52 \mathrm{~mm}$, for right and left ankle, respectively. Coefficients of variation for repeat measurements by the same observer were $1.13 \%$ and $4.5 \%$, and by different observers $7.30 \%$ and $7.27 \%$, for medial and lateral joint space widths, respectively. Men had wider joint space widths than women when accounting for height. Joint space width of the ankle correlated with the joint space width of the hip and with height and weight, but not with age.

\section{Introduction}

Osteoarthritis (OA) of the ankle is less common than OA of either the hip or knee, but it nonetheless represents a substantial clinical problem, especially after trauma $[4,19]$. In large series, trauma is the most common etiologic factor and primary (idiopathic) OA accounts for less than $10 \%$ of ankle OA [4, 23].

In the knee and hip, quantification of the radiographic joint space width (JSW) represents a primary means of assessing OA severity and progression [10, 14, 18]. However, in the ankle, radiographic evaluation often uses nonquantitative grading systems [20, 22, 24], which are subjective and may suffer from reliability and reproducibility problems. For example, a nonquantitative grading system for ankle OA, described by van Dijk et al. [24] in a study investigating outcomes of arthroscopic surgery for anterior ankle impingement, involves four grades (Grades $0-3$ ) ranging from Grade 0 for a normal joint to Grade 3 for 
(sub)total disappearance/deformation of the joint space. However, reproducibility and reliability for this method have not been reported. Similarly, Tallroth et al. [22] used a comparable nonquantitative grading system for their study of ankles in patients with knee OA, which ranged from Grade 0 for normal joints to Grade 2 for marked or severely narrowed joint space, sclerotic changes, and marked osteophytes. Again, reproducibility and reliability were not reported. As in the case of the qualitative grading systems for assessing ankle JSW, there have been relatively few quantitative methods specifically developed for the ankle reported to date, and those have not been well validated $[8,9,12]$. The studies by Jonsson et al. [12] and Fars $\varnothing$ Nielsen and de Carvalho [8] measured JSW directly on radiographs (tibiotalar compartment) at three locations (medial, central, and lateral) and defined the joint space as the distance between the cortices of the tibia and the talus, perpendicular to the joint surface; neither provided details of their methodology or any reproducibility analyses. One method, described by Marijnissen et al. [16], used proprietary software to analyze ankle JSW quantitatively. This system, the "ankle image digital analysis" (AIDA) [16], requires the operator to mark three lines to define the borders of the joint space; the program then automatically divides the joint space by drawing five lines perpendicular to the talus bone cartilage interface and yields JSW information. Although this semiautomatic digital method has good reproducibility, it requires specific software not readily available.

Although radiographic evaluation has its inherent limitations owing to its projectional technique and does not allow direct observation of the cartilage, quantitative assessment of radiographic JSW provides important diagnostic and prognostic information $[5,7,10]$. In OA of the knee and hip, JSW determinations relate to OA severity [5, $7,14,18]$ and reflect the only structural outcome currently accepted by the US FDA for trials of potential OA diseasemodifying agents. To advance the understanding of ankle $\mathrm{OA}$, it also is important to identify convenient and reliable methods to quantify JSW of the ankle. In addition, as radiographic JSW measurements may be interpreted prognostically, their relationship to normal body variation must be defined. We previously observed JSW of the hip is seemingly narrower in women than in men, although this relationship is lost after correction for height [11]. In addition, JSW of the hip is correlated with height and weight, but not with body mass index (BMI).

The purposes of our study were (1) to determine the reliability of a new method to quantify the radiographic JSW of the ankle using software that is free and widely available, and (2) to assess the relationship between JSW of the ankle and gender, weight, height, and BMI, and to evaluate potential correlations with JSW of the hip.

\section{Materials and Methods}

We recruited 95 patients (71 females, 24 males) with symptomatic medial knee OA into a 36-month longitudinal study of knee OA (ClinicalTrials.gov, NCT00076453). Enrollment criteria were described previously [17], but briefly, patients were included if they fulfilled the American College of Rheumatology criteria for the classification of knee OA [1], with at least $20 \mathrm{~mm}$ (on a 100-mm visual analog scale) of knee pain while walking on a level surface; had radiographic OA of Kellgren-Lawrence Grades 2 or 3 [13]; and had predominantly medial compartment involvement. We excluded patients who had clinically evident OA involving lower extremity joints other than the knees, any inflammatory arthropathy, a BMI greater than $35 \mathrm{~kg} / \mathrm{m}^{2}$, a history of fracture of either lower extremity within 6 months of study entry, or prior arthroplasty of any lower extremity joint. At the screening visit, all subjects were evaluated by a podiatrist and we further excluded patients with evidence of intrinsic foot or ankle disease, such as hallux rigidus, hallux abductovalgus, metatarsalgia, plantar fasciitis, or peripheral neuropathy. Each subject completed a site-directed WOMAC pain questionnaire [2] for each lower extremity joint, and those who noted greater than a total of $20 \mathrm{~mm}$ (of a total $500 \mathrm{~mm}$ possible) on a pain visual analog scale for the feet and ankles were excluded. Gender, age, weight, and height data were recorded. All patients provided informed consent for participation in the study.

At baseline, each patient had weightbearing digital anteroposterior (AP) radiographs of the ankle and supine AP radiographs of the pelvis. We quantified the narrowest JSWs of the hips and ankles on the digital images using Image J software (US NIH, Bethesda, MD, http://rsb.info. nih.gov/ij/). The JSW of the hip was defined as the narrowest point between the cortical surface of the acetabulum and the bone contour of the femoral head [10]. The Image $\mathbf{J}$ software was adapted to digital radiographic images as follows: the narrowest apparent distance was visually selected and a line connecting each opposing bone cortical surface was produced. The line then was moved digitally through the entire joint space to confirm it represented the narrowest JSW; if the operator identified a point in the joint space where the line was too long, a new line was produced at that point. When the entire joint space had been assessed, the length of the resulting line was quantified by Image J, which was defined as the radiographic JSW. For the ankles, the midpoint of the upper surface of the talus was determined using the Image $\mathbf{J}$ software as follows: the medial and lateral edges of the talus were marked, a line parallel to the surface was produced, and the midpoint was determined by the software. The JSW was defined as the narrowest distance between the upper surface of the talus and the distal 


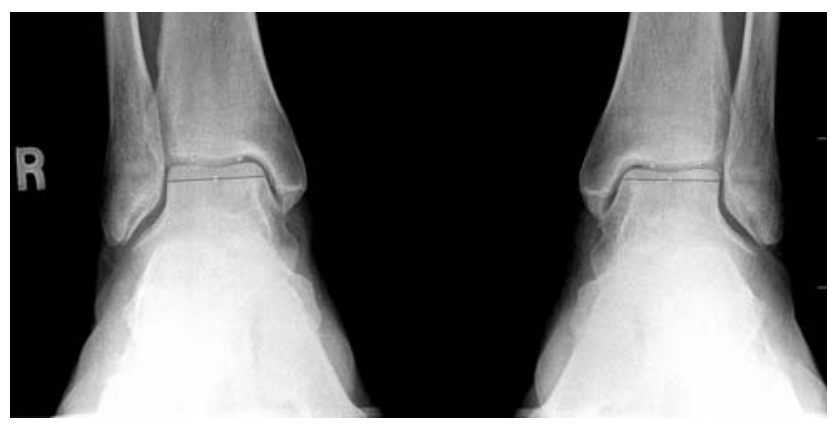

Fig. 1 The midpoint of the upper surface of the talus was used to separate the medial joint space of the ankle from lateral. The narrowest distance between the upper surface of the talus and the distal end of the tibia was measured on the medial and lateral sides of this midpoint. This distance was not necessarily perpendicular to any bony surface. The narrower of the medial or lateral JSW was taken as the narrowest JSW of the ankle.

end of the tibia and was determined for the medial and lateral sides of this midpoint, using the described method (Fig. 1). This was not necessarily perpendicular to any landmark. For assigning one JSW for the tibiotalar joint, the narrower of the medial or lateral JSW was taken as the JSW of the ankle.

All assessments were performed with the evaluators blinded to subject identity and to prior assessments, including blinding to prior landmark identifications. Fifteen ankles were evaluated twice by two observers (BG, EG) for interobserver and intraobserver variability. The repeat JSW assessments were performed 3 to 7 days after the first readings and the evaluators were blinded to the previous results.

For intraobserver and interobserver reproducibility of the measurements, coefficients of variation analysis were performed. For each of the repeated measurements $(\mathrm{n}=15)$, (standard deviation $[\mathrm{SD}] /$ mean $) \times 100$ was calculated and the mean of these was taken as the coefficient of variation. The degree of agreement between repeated measurements by one observer and by separate observers also was analyzed by the limits of agreement method described by Bland and Altman [3]. The narrowest JSWs were normally distributed (one-sample KolmogorovSmirnov test, $\mathrm{p}>0.05$ for all). A limits of agreement analysis provides an estimate of how closely the two measurements track and is based on the expectation that if the measurement differences are normally distributed, $95 \%$ of the differences will be found in the range between the mean \pm 1.96 SD. The minimum detectable change, defined as 1.96 times the SD of the differences between repeated measurements, also was determined.

Using Pearson's correlation analyses, we explored any relationships between the most narrow recorded JSW of the ankles and JSW of the hips and between the most narrow recorded JSW of the ankle and anthropometric characteristics, including height, weight, and BMI. We compared the mean medial and lateral JSWs of the ankles using a paired-sample $t$ test. Mean JSWs of ankles of men and women were compared using Student's t test. Multiple regression analysis was performed to evaluate the effect of gender and body height (as independent variables) on the JSW. Statistical analyses were performed using SPSS $^{\circledR}$ 11.0 software (SPSS Inc, Chicago, IL).

\section{Results}

Intraobserver coefficients of variations for medial and lateral JSW measurements were $1.13 \%$ and $4.5 \%$, respectively (Table 1). Interobserver coefficients of variations for medial and lateral JSW measurements were $7.30 \%$ and $7.27 \%$, respectively. Limits of agreement for repeat measurements by the same observer were $-0.11 \mathrm{~mm}$ to $0.16 \mathrm{~mm}$ for the medial JSW (Fig. 2A) and $-0.34 \mathrm{~mm}$ to $0.28 \mathrm{~mm}$ for the lateral JSW (Fig. 2B) of the ankle. The minimum detectable change was $0.14 \mathrm{~mm}$ for the medial JSW and $0.31 \mathrm{~mm}$ for the lateral JSW of the ankle. Limits of agreement for measurements made by two different observers were $-0.17 \mathrm{~mm}$ to $0.58 \mathrm{~mm}$ for the medial JSW (Fig. 3A) and -0.52 to $0.62 \mathrm{~mm}$ for the lateral JSW of the ankle (Fig. 3B). The minimum detectable change was $0.38 \mathrm{~mm}$ for the medial JSW and $0.57 \mathrm{~mm}$ for the lateral JSW of the ankle.

The JSW of the ankle correlated with the JSW of the hip, and with height and weight, but not with age or BMI (Table 2). Medial and lateral JSWs of the ankle correlated individually with the JSWs of the hip (Table 3). Men had wider JSWs than women at all measurement sites

Table 1. Results of intraobserver and interobserver variability tests (15 ankles)

\begin{tabular}{|c|c|c|c|c|}
\hline \multirow[t]{2}{*}{ Analysis } & \multicolumn{2}{|l|}{ Intraobserver } & \multicolumn{2}{|l|}{ Interobserver } \\
\hline & Medial JSW & Lateral JSW & Medial JSW & Lateral JSW \\
\hline Coefficients of variation $(\%)$ & 1.13 & 4.5 & 7.30 & 7.27 \\
\hline Limits of agreement (mm) & -0.11 to 0.16 & -0.34 to 0.28 & -0.17 to 0.58 & -0.52 to 0.62 \\
\hline Minimum detectable change $(\mathrm{mm})$ & 0.14 & 0.31 & 0.38 & 0.57 \\
\hline
\end{tabular}

JSW = joint space width. 

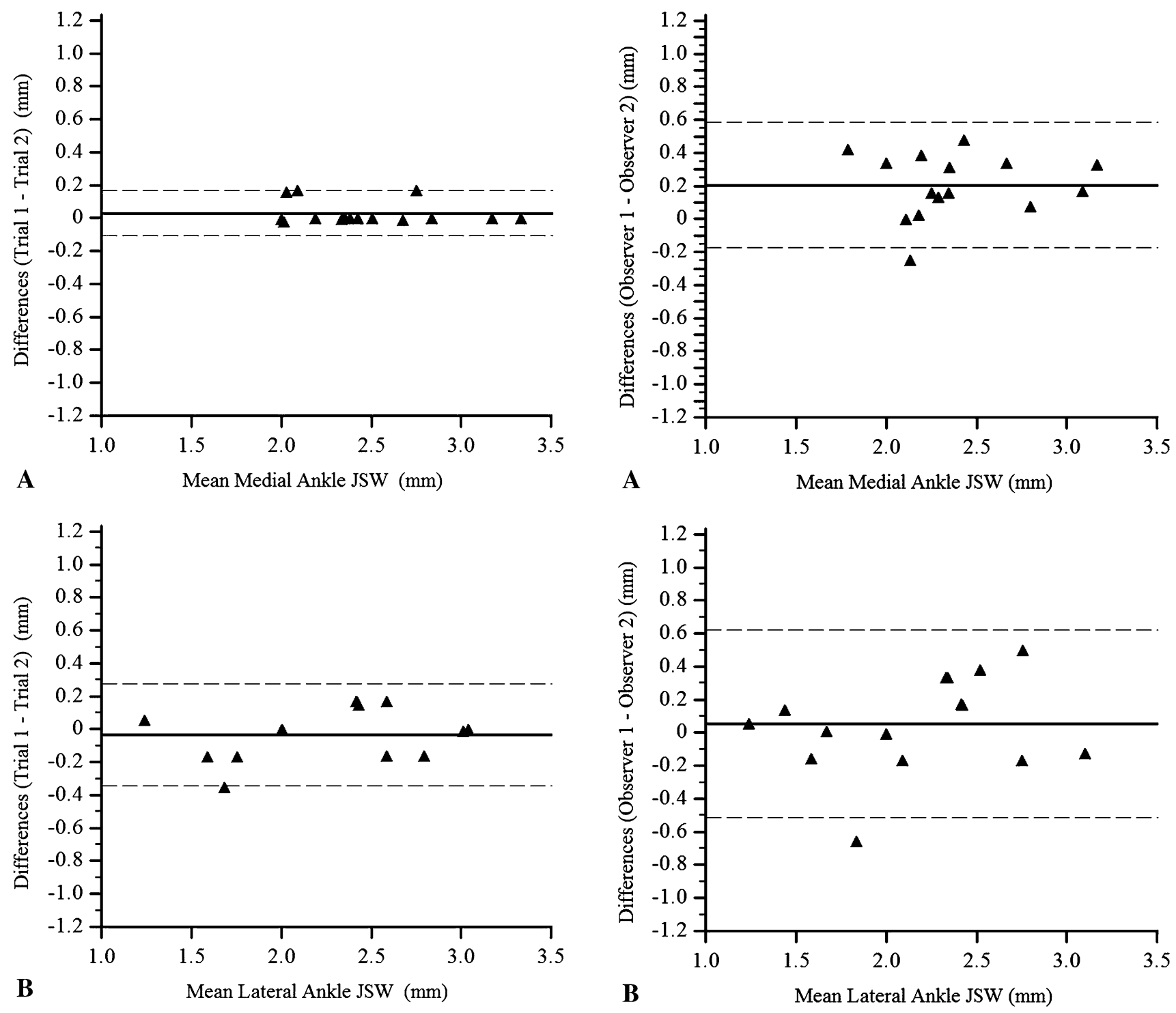

Fig. 2A-B Agreement between repeat measurements by the same observer for the narrowest (A) medial JSW and (B) lateral JSW of the ankle, using the method of Bland and Altman [2], is shown. Each graph illustrates the limits of agreement. The solid lines delineate the mean differences, and the dotted lines represent the mean difference $\pm 1.96 \mathrm{SD}$. The mean \pm SD differences between the repeated measurements were $+0.029 \pm 0.069 \mathrm{~mm}$ for the medial JSW and $-0.033 \pm 0.159 \mathrm{~mm}$ for the lateral JSW.

(Table 4), and when gender and height were taken as independent variables, male gender was a predictor for wider JSW at all sites, but height was not (Table 5). The medial joint spaces were wider than the lateral joint spaces for right and left ankles (Table 6).

\section{Discussion}

OA of the ankle is a major clinical problem, including after trauma $[4,19]$. Quantitative evaluation of changes in the

Fig. 3A-B Agreement between measurements by two observers for the narrowest (A) medial JSW and (B) lateral JSW of the ankle, using the method of Bland and Altman [2], is shown. Each graph illustrates the limits of agreement. The solid lines delineate the mean differences, and the dotted lines represent the mean difference \pm 1.96 $\mathrm{SD}$. The mean $\pm \mathrm{SD}$ differences between the measurements were $+0.21 \pm 0.19 \mathrm{~mm}$ for the medial JSW and $0.05 \pm 0.29$ for the lateral JSW.

radiographic joint space is the standard by which structural progression in OA may be assessed, yet currently available techniques for measuring the joint space of the ankle either require proprietary software or have not been well validated $[8,12,16]$. A method that would reliably and conveniently determine the JSW using standard clinical radiographs of the ankle could provide information essential to evaluate the effects of potential disease-modifying agents and would permit monitoring the ankles for potential adverse effects of devices intended to ameliorate OA elsewhere in the lower extremity. Therefore we describe a simple and reproducible 
Table 2. Correlations between JSW of the ankle and anthropometric characteristics

\begin{tabular}{lcr}
\hline Correlation $(\mathrm{n}=95)$ & $\mathrm{r}$ Value & $\mathrm{p}$ Value \\
\hline $\begin{array}{l}\text { Ipsilateral JSW of the hip } \\
\text { JSW of the right ankle }\end{array}$ & 0.40 & $<0.001$ \\
JSW of the left ankle & 0.49 & $<0.001$ \\
Height & 0.30 & 0.003 \\
JSW of the right ankle & 0.42 & $<0.001$ \\
JSW of the left ankle & & 0.03 \\
Weight & 0.22 & 0.04 \\
JSW of the right ankle & 0.22 & 0.41 \\
JSW of the left ankle & & 0.83 \\
Body mass index & 0.09 & \\
JSW of the right ankle & 0.02 & 0.98 \\
JSW of the left ankle & & 0.32 \\
Age & -0.003 & -0.10 \\
$\quad$ JSW of the right ankle & & \\
JSW of the left ankle &
\end{tabular}

$\mathrm{JSW}=$ joint space width.

Table 3. Correlations between JSWs of the ankle and ipsilateral hip

\begin{tabular}{lll}
\hline Correlation $(\mathrm{n}=95)$ & \multicolumn{2}{l}{ JSW of the ipsilateral hip } \\
\cline { 2 - 3 } & $\mathrm{r}$ Value & $\mathrm{p}$ Value \\
\hline Right ankle & 0.37 & $<0.001$ \\
$\quad$ Medial JSW & 0.34 & $<0.001$ \\
$\quad$ Lateral JSW & & \\
Left ankle & 0.48 & $<0.001$ \\
$\quad$ Medial JSW & 0.43 & $<0.001$ \\
Lateral JSW & &
\end{tabular}

$\mathrm{JSW}=$ joint space width .

Table 4. Gender differences in JSWs of the ankle

\begin{tabular}{llll}
\hline JSW/Ankle & JSW $(\mathrm{mm})$ & p Value \\
\cline { 2 - 3 } & Men $(\mathrm{n}=24)$ & Women $(\mathrm{n}=71)$ & \\
\hline Medial JSW & & & \\
$\quad$ Right ankle & $3.02 \pm 0.38$ & $2.40 \pm 0.43$ & $<0.001$ \\
$\quad$ Left ankle & $2.97 \pm 0.51$ & $2.28 \pm 0.45$ & $<0.001$ \\
Lateral JSW & & & \\
$\quad$ Right ankle & $3.00 \pm 0.44$ & $2.40 \pm 0.39$ & $<0.001$ \\
$\quad$ Left ankle & $2.86 \pm 0.44$ & $2.30 \pm 0.46$ & $<0.001$ \\
Narrowest JSW* & & & \\
$\quad$ Right ankle & $2.81 \pm 0.38$ & $2.22 \pm 0.43$ & $<0.001$ \\
$\quad$ Left ankle & $2.79 \pm 0.41$ & $2.20 \pm 0.44$ & $<0.001$ \\
\hline
\end{tabular}

Values are expressed as mean \pm standard deviation; $*$ narrower of the medial or lateral JSW taken as the narrowest JSW of the ankle; $\mathrm{JSW}=$ joint space width. method to quantify the radiographic JSW of the ankle. We first determined the reliability of this method and then used it to assess the relationships between JSW of the ankle and gender, weight, height, and BMI, and with the JSW of the hip.

The major limitation of this study is that the study population was comprised of patients with knee OA rather than healthy subjects, raising the possibility that the measured JSWs may not be representative of the general population. However, anyone with clinically detectable pain or disease of the ankle, foot, or hip was excluded from the study. Moreover, as the ankle usually is spared in primary OA, we believe it is likely these data reflect those of a healthy population. A second limitation of the study is that test-retest variability could not be confirmed, ie, ankle radiographs were not repeated at short intervals to assess the variability related to repeat radiography; this step was not included in the protocol of primary study to reduce radiation exposure. A third limitation is we had no way to assess accuracy of the measurements and therefore could not validate the technique. Nonetheless, the technique is analogous to those used for other joints and is reliable.

Nonquantitative grading systems for evaluation of radiographic features of $\mathrm{OA}$ are subjective and, as discussed previously, may suffer from problems with reliability and reproducibility. Quantitative assessments of the radiographic JSWs of the knee and hip have become standard outcome measures for studies evaluating the pathophysiologic mechanisms or epidemiology of OA, and potential disease-modifying agents. In contrast, quantitative assessment of the JSW of the ankle has been less well studied and is not yet considered a conventional outcome parameter. Currently, the optimal method remains unclear; prior studies used conventional radiographs [12] or complex systems not widely available [16]. The software used for the methodology described in the current study is open access and therefore free and readily available. Reproducibility analyses show this method results in better intraobserver and similar interobserver variabilities compared with the previously described AIDA method (Table 7) [16]. The AIDA method used mean JSWs (the mean of five measurements in each ankle), whereas our method focuses on the narrowest JSW of each ankle, which reflects the standard used in other joints [15, 21]. OA tends to affect joints asymmetrically, ie, predominantly medially or laterally. Therefore, the overall mean JSWs across the entire joint may be less sensitive compared with the narrowest JSW. In the Image $\mathrm{J}$ method, the reproducibility of results was poorer for lateral JSWs compared with medial JSWs. This may relate to the fact that lateral JSWs are substantially narrower than the medial ones or than the less homogeneous radiographic appearance of the bony landmarks on the lateral aspect of the tibiotalar joint. 
Table 5. Effects of gender and height on JSWs of the ankle

\begin{tabular}{|c|c|c|c|c|c|c|}
\hline \multirow[t]{2}{*}{ Correlation $(\mathrm{n}=95)$} & \multicolumn{3}{|c|}{ Male gender } & \multicolumn{3}{|l|}{ Height } \\
\hline & $\mathrm{b}$ & SE & $\mathrm{t}$ value/p value & $\mathrm{b}$ & SE & $\mathrm{t}$ value/p value \\
\hline \multicolumn{7}{|l|}{ Medial JSW } \\
\hline Right ankle & 0.581 & 0.132 & $4.39 /<0.001$ & 0.002 & 0.006 & $0.38 / 0.70$ \\
\hline Left ankle & 0.413 & 0.123 & $3.37 / 0.001$ & 0.013 & 0.006 & $2.33 / 0.022$ \\
\hline \multicolumn{7}{|l|}{ Lateral JSW } \\
\hline Right ankle & 0.780 & 0.146 & $5.34 /<0.001$ & -0.006 & 0.007 & $-0.88 / 0.38$ \\
\hline Left ankle & 0.496 & 0.142 & $3.49 / 0.001$ & 0.004 & 0.006 & $0.65 / 0.52$ \\
\hline \multicolumn{7}{|l|}{ Narrowest JSW* } \\
\hline Right ankle & 0.658 & 0.131 & $5.02 /<0.001$ & -0.004 & 0.006 & $-0.72 / 0.47$ \\
\hline Left ankle & 0.506 & 0.146 & $3.48 / 0.001$ & 0.004 & 0.007 & $0.66 / 0.51$ \\
\hline
\end{tabular}

* Narrower of the medial or lateral JSW taken as the narrowest ankle JSW; $b=$ regression coefficient; SE = standard error; JSW = joint space width.

Table 6. Medial and lateral JSWs of the ankle

\begin{tabular}{llll}
\hline Ankle & \multicolumn{2}{l}{ Narrowest ankle JSW $(\mathrm{mm})(\mathrm{n}=95)$} & $\mathrm{p}$ Value \\
\cline { 2 - 3 } & Medial & \multicolumn{2}{c}{ Lateral } \\
\hline Right & $2.56 \pm 0.5(1.29-3.91)$ & $2.45 \pm 0.55(1.36-4.12)$ & 0.012 \\
Left & $2.55 \pm 0.48(1.25-4.46)$ & $2.44 \pm 0.52(1.30-3.94)$ & 0.004
\end{tabular}

Values are expressed as mean \pm standard deviation, with range in parentheses; JSW = joint space width.

One reason for the absence of standardized methodology for determining the radiographic JSW of the ankle may be the relatively uncommon nature of ankle OA, and therefore, of clinically apparent degenerative processes of the ankle. However, OA of the ankle resulting from trauma is a substantial problem among competitive athletes and may be expected to increase as an aging population stays athletically active longer. Saltzman et al. [19] reported $7.2 \%$ of ankle arthritis is primary and half of these patients do not have identified predisposing foot deformities. The average age of onset of primary OA in their study was 67.2 years, which is substantially older than is seen with other causes of ankle arthritis. In addition to clinically evident arthritis, degeneration of ankle cartilage is common among the elderly [6]. Thus, it may be expected, as the population of elderly increases during the next few decades and as the elderly remain physically active longer, ankle OA may become an increasingly important clinical problem.

Our findings that the medial joint space is wider than the lateral joint space in ankles and that men have wider joint spaces than women are consistent with previous reports using conventional radiographs [8, 12]. However, those studies used the mean JSW rather than the narrowest JSW to define the ankle JSW; therefore, direct comparisons are not appropriate. We also found male gender was a
Table 7. Reproducibility of comparative studies on quantification of JSW of the ankle

\begin{tabular}{|c|c|c|c|}
\hline \multirow[t]{2}{*}{ Study } & \multirow[t]{2}{*}{ JSW } & \multicolumn{2}{|c|}{ Limits of agreement analysis } \\
\hline & & Intraobserver & Interobserver \\
\hline $\begin{array}{l}\text { Marijnissen } \\
\text { et al. [16] }\end{array}$ & Mean JSW & -0.45 to 0.43 & -0.66 to 0.50 \\
\hline \multirow[t]{2}{*}{ Current study } & $\begin{array}{l}\text { Medial narrowest } \\
\text { JSW }\end{array}$ & -0.11 to 0.16 & -0.17 to 0.58 \\
\hline & $\begin{array}{l}\text { Lateral narrowest } \\
\text { JSW }\end{array}$ & -0.34 to 0.28 & -0.52 to 0.62 \\
\hline
\end{tabular}

$\mathrm{JSW}=$ joint space width.

predictor for wider JSW at all sites. The results suggest the difference is independent of height. In addition, perhaps unsurprisingly but not reported previously, the JSW of the ankle appears to correlate well with the JSWs of other clinically normal lower extremity joints, such as the hips, and with height.

We have proposed a new method to quantify radiographic JSW of the ankle. The radiographic JSW of the ankle can be reliably and conveniently quantified using Image J. This method might facilitate studies investigating cartilage biology or biomechanics when reliable radiographic evaluations are needed.

\section{References}

1. Altman R, Asch E, Bloch D, Bole G, Borenstein D, Brandt K, Christy W, Cooke TD, Greenwald R, Hochberg M, et al. Development of criteria for the classification and reporting of osteoarthritis: classification of osteoarthritis of the knee. Diagnostic and Therapeutic Criteria Committee of the American Rheumatism Association. Arthritis Rheum. 1986;29:1039-1049.

2. Bellamy N, Buchanan WW, Goldsmith CH, Campbell J, Stitt LW. Validation study of WOMAC: a health status instrument for measuring clinically important patient relevant outcomes to 
antirheumatic drug therapy in patients with osteoarthritis of the hip or knee. J Rheumatol. 1988;15:1833-1840.

3. Bland JM, Altman DG. Statistical methods for assessing agreement between two methods of clinical measurement. Lancet. 1986;1:307-310.

4. Brown TD, Johnston RC, Saltzman CL, Marsh JL, Buckwalter JA. Posttraumatic osteoarthritis: a first estimate of incidence, prevalence, and burden of disease. $J$ Orthop Trauma. 2006;20:739-744.

5. Bruyere O, Richy F, Reginster JY. Three year joint space narrowing predicts long term incidence of knee surgery in patients with osteoarthritis: an eight year prospective follow up study. Ann Rheum Dis. 2005;64:1727-1730.

6. Cole AA, Margulis A, Kuettner KE. Distinguishing ankle and knee articular cartilage. Foot Ankle Clin. 2003;8:305-316.

7. Dougados M, Gueguen A, Nguyen M, Berdah L, Lequesne M, Mazieres B, Vignon E. Radiological progression of hip osteoarthritis: definition, risk factors and correlations with clinical status. Ann Rheum Dis. 1996;55:356-362.

8. Fars $\varnothing$ Nielsen F, de Carvalho A. Dependence of ankle joint width on plantar flexion. Acta Radiol. 1989;30:554-556.

9. Fiirgaard B, Iversen JK, de Carvalho A. Width of the medial tibiotalar joint. Acta Radiol. 1997;38:520-522.

10. Goker B, Doughan AM, Schnitzer TJ, Block JA. Quantification of progressive joint space narrowing in osteoarthritis of the hip: longitudinal analysis of the contralateral hip after total hip arthroplasty. Arthritis Rheum. 2000;43:988-994.

11. Goker B, Sancak A, Arac M, Shott S, Block JA. The radiographic joint space width in clinically normal hips: effects of age, gender and physical parameters. Osteoarthritis Cartilage. 2003;11:328334.

12. Jonsson K, Fredin HO, Cederlund CG, Bauer M. Width of the normal ankle joint. Acta Radiol Diagn (Stockh). 1984;25:147-149.

13. Kellgren JH, Lawrence JS. Radiological assessment of osteoarthrosis. Ann Rheum Dis. 1957;16:494-502.

14. Lane NE, Nevitt MC, Hochberg MC, Hung YY, Palermo L. Progression of radiographic hip osteoarthritis over eight years in a community sample of elderly white women. Arthritis Rheum. 2004;50:1477-1486.
15. Maillefert JF, Nguyen M, Gueguen A, Berdah L, Lequesne M, Mazières B, Vignon E, Dougados M. Relevant change in radiological progression in patients with hip osteoarthritis. II. Determination using an expert opinion approach. Rheumatology (Oxford). 2002;41:148-152.

16. Marijnissen AC, Vincken KL, Viergever MA, van Roy HL, Van Roermund PM, Lafeber FP, Bijlsma JW. Ankle images digital analysis (AIDA): digital measurement of joint space width and subchondral sclerosis on Standard radiographs. Osteoarthritis Cartilage. 2001;9:264-272.

17. Mody S, Jolly M, Kwasny MJ, Block JA. Patient reported outcomes and analgesia use in osteoarthritis of the knee. Osteoarthritis Cartilage. 2008;16:1294-1299.

18. Nevitt MC, Peterfy C, Guermazi A, Felson DT, Duryea J, Woodworth T, Chen H, Kwoh K, Harris TB. Longitudinal performance evaluation and validation of fixed-flexion radiography of the knee for detection of joint space loss. Arthritis Rheum. 2007;56:1512-1520.

19. Saltzman CL, Salamon ML, Blanchard GM, Huff T, Hayes A, Buckwalter JA, Amendola A. Epidemiology of ankle arthritis: report of a consecutive series of 639 patients from a tertiary orthopaedic center. Iowa Orthop J. 2005;25:44-46.

20. Schmitt H, Lemke JM, Brocai DR, Parsch D. Degenerative changes in the ankle in former elite high jumpers. Clin J Sport Med. 2003;13:6-10.

21. Sharma L, Lou C, Cahue S, Dunlop DD. The mechanism of the effect of obesity in knee osteoarthritis: the mediating role of malalignment. Arthritis Rheum. 2000;43:568-575.

22. Tallroth K, Harilainen A, Kerttula L, Sayed R. Ankle osteoarthritis is associated with knee osteoarthritis: conclusions based on mechanical axis radiographs. Arch Orthop Trauma Surg. 2008;128:555-560.

23. Valderrabano V, Horisberger M, Russell I, Dougall H, Hintermann B. Etiology of Ankle Osteoarthritis. Clin Orthop Relat Res. 2008 Oct 2. [Epub ahead of print].

24. van Dijk CN, Tol JL, Verheyen CC. A prospective study of prognostic factors concerning the outcome of arthroscopic surgery for anterior ankle impingement. Am J Sports Med. 1997;25:737-745. 